\title{
REGULARITY PROPERTIES OF ATTAINABLE SETS UNDER STATE CONSTRAINTS
}

\author{
P. CANNARSA ${ }^{\dagger}$ and M. CASTELPIETRA ${ }^{\ddagger}$ \\ Dipartimento di Matematica, Università di Roma Tor Vergata, \\ 00133 Roma, Italia \\ ${ }^{\dagger}$ E-mail: cannarsa@mat.uniroma2.it \\ ${ }^{\ddagger}$ E-mail: castelpi@mat.uniroma2.it \\ http://www.mat.uniroma2.it \\ P. CARDALIAGUET \\ Laboratoire de Mathématiques, Université de Bretagne Occidentale \\ 29285 Brest, France \\ E-mail: Pierre.Cardaliaguet@univ-brest.fr \\ http://maths2.univ-brest.fr
}

\begin{abstract}
The Maximum principle in control theory provides necessary optimality conditions for a given trajectory in terms of the co-state, which is the solution of a suitable adjoint system. For constrained problems the adjoint system contains a measure supported at the boundary of the constraint set. In this paper we give a representation formula for such a measure for smooth constraint sets and nice Hamiltonians. As an application, we obtain a perimeter estimate for constrained attainable sets.
\end{abstract}

Keywords: Maximum principle; Interior sphere property; Perimeter.

\section{Introduction}

The Maximum principle is a fundamental result in optimal control theory. Not only does it provide necessary conditions for candidate solutions of an optimal control problem, but it can also be used to obtain regularity results for optimal trajectories.

To fix ideas, consider a control system of the form

$$
\left\{\begin{array}{l}
x^{\prime}(t)=f(t, x(t), u(t)), \quad u(t) \in U \quad \text { a.e. } t \geq 0 \\
x(0)=x_{0}
\end{array}\right.
$$

where $u(\cdot)$ is a control, and $x(\cdot)$ denotes the corresponding trajectory. 
Consider an open set $\Omega \subseteq \mathbb{R}^{n}$. For a point $x_{0} \in \Omega$ we say that a control $u(\cdot)$ is admissible in $x_{0}$ on $[0, T]$ if

$$
x(t) \in \bar{\Omega} \quad \forall t \in[0, T],
$$

where $x(\cdot)$ is the corresponding (then, admissible) trajectory. $\bar{\Omega}$ is called the constraint set.

Denote by $\mathcal{A}(t)$ the attainable set in time $t$ from a closed set $\mathcal{K} \subseteq \Omega$. We call optimal an admissible trajectory $x(\cdot)$ on the interval $[0, T]$ if $T=$ $\inf \{t \geq 0: x(T) \in \mathcal{A}(t)\}$, that is, if the trajectory $x(\cdot)$ minimizes the time to reach the point $x(T)$.

Every optimal trajectory satisfies the Maximum Principle: this result was obtained by several authors in the smooth case, ${ }^{8,10,12}$ and, as a natural evolution, also in the nonsmooth case..$^{2,7,9,11,13}$

In constrained problems there are singularity effects for the trajectories that touch the boundary of $\Omega$. These effects are due to an additional term (containing a measure) that appears in the Maximum Principle. More precisely, introducing the Hamiltonian

$$
H(t, x, p)=\sup _{u \in U}\langle f(t, x, u), p\rangle,
$$

we have that, if $x(\cdot)$ is an optimal trajectory, then there exist an $\operatorname{arc} p(\cdot)$ and a Radon measure $\mu$ such that for a.e. $t \geq 0$

$$
\left(h^{\prime}(t),-p^{\prime}(t), x^{\prime}(t)\right) \in \partial H(t, x(t), p(t)+\psi(t)),
$$

where

$$
\begin{aligned}
& \psi(t)=\int_{[0, t)} \nu_{\Omega}(x(s)) \mu(d s), \\
& h(t)=H(t, x(t), p(t)+\psi(t)) .
\end{aligned}
$$

Here, $\nu_{\Omega}(x)$ belongs to the normal cone to $\Omega$ at $x$, and the measure $\mu$ is supported only by the set of times $t$ for which $x(t) \in \partial \Omega$ (see Ref. 7).

If we have a "nice" Hamiltonian (in Sec. 2 we clarify the meaning of nice), then the generalized gradient of $H$ turns to be the triplet $\left(D_{t} H, D_{x} H, D_{p} H\right)$, and inclusion (2) becomes an equality.

The main result of this paper, developed in the next section, gives a representation of the additional term $\psi$ in the Maximum Principle. That is, we show that, if $\partial \Omega$ is smooth, then $x^{\prime}(t)=D_{p} H(t, x(t), p(t))$ and

$$
-p^{\prime}(t)=D_{x} H(t, x(t), p(t))-\lambda(t) \nu_{\Omega}(x(t)) \mathbf{1}_{\partial \Omega}(x(t)),
$$

where $\lambda$ is a measurable function, depending on $\partial \Omega$ and $H$, that we explicitly compute. 
In the last section of this paper, we apply the above formulation of the Maximum Principle to extend a result by Cardaliaguet and $\mathrm{Marchi}^{6}$ for perimeters of attainable sets.

\section{Maximum principle under state constraints}

Let $\Omega \subseteq \mathbb{R}^{n}$ be an open set with uniformly $\mathcal{C}^{2}$ boundary. We define the signed distance from $\partial \Omega$ by

$$
d(x)=d_{\Omega}(x)-d_{\Omega^{c}}(x) \quad x \in \mathbb{R}^{n},
$$

where we have denoted by $d_{S}(x)=\inf \{|x-y|: y \in S\}$ the distance function from a set $S \subseteq \mathbb{R}^{n}$. The boundary of $\Omega$ is of class $\mathcal{C}^{2}$ if and only if there exists some $\eta>0$ such that

$$
d(\cdot) \in \mathcal{C}_{\mathrm{b}}^{2} \text { on } \partial \Omega+B_{\eta}=\{y \in B(x, \eta): x \in \partial \Omega\},
$$

where $\mathcal{C}_{\mathrm{b}}^{2}$ is the set of functions of class $\mathcal{C}^{2}$ with bounded derivatives of first and second order.

We consider the following controlled system, subject to state constraints,

$$
\left\{\begin{array}{l}
x^{\prime}(t)=f(t, x(t), u(t)), \quad \text { a.e. } t \geq 0 \\
x(t) \in \bar{\Omega}, \quad t \geq 0 \\
x(0)=x_{0},
\end{array}\right.
$$

where $U \subseteq \mathbb{R}^{m}$ is a compact set, and $u: \mathbb{R}_{+} \rightarrow U$ is measurable function, in short an admissible control. We assume that $f: \mathbb{R}_{+} \times \mathbb{R}^{n} \times U$ is a continuous function such that, for some positive constants $L$ and $k$,

$$
\left\{\begin{array}{l}
|f(t, x, u)-f(t, y, u)| \leq L|x-y| \quad \forall x, y \in \mathbb{R}^{n}, \forall(t, u) \in \mathbb{R}_{+} \times U \\
|f(t, x, u)| \leq k(1+|x|) \quad \forall(t, x, u) \in \mathbb{R}_{+} \times \mathbb{R}^{n} \times U
\end{array}\right.
$$

Let $\mathcal{K} \subseteq \Omega$ be a given closed set. The set of admissible trajectories from $\mathcal{K}($ at time $t)$ is

$$
\mathcal{T}_{\text {ad }}(\mathcal{K}, t)=\left\{\text { trajectories } x(\cdot) \text { that solve }(4) \text { in }[0, t] \text {, such that } x_{0} \in \mathcal{K}\right\} .
$$

The attainable set from $\mathcal{K}$ at time $t$ is defined by

$$
\mathcal{A}(\mathcal{K}, t)=\left\{x(t): x(\cdot) \in \mathcal{T}_{\text {ad }}(\mathcal{K}, t)\right\} .
$$

We introduce the minimum time function

$$
\tau_{\mathcal{K}}(x)=\inf \{t: x \in \mathcal{A}(\mathcal{K}, t)\},
$$

that is the time needed to reach a point $x$, starting from $\mathcal{K}$.

For state constrained systems the definition of "extremal solutions" differs, somewhat, from the unconstrained case. In fact, it is not sufficient to 
request that trajectories be on the boundary of $\mathcal{A}(\mathcal{K}, t)$, because a trajectory can stay on the boundary of $\Omega$ without being "optimal".

Example 2.1. Take $\Omega=\left\{x \in \mathbb{R}^{2}:\left\langle x, \mathbf{e}_{2}\right\rangle<1\right\}$, where $\left\{\mathbf{e}_{1}, \mathbf{e}_{2}\right\}$ is an orthonormal basis of $\mathbb{R}^{2}$. Let $\mathcal{K}=\{0\}$, and $f(t, x, u)=u$ with $U=\bar{B}$, and $T=3$.

Consider the trajectories $x_{1}(\cdot)$ and $x_{2}(\cdot)$ associated, respectively, to the controls

$$
u_{1}(t)=\mathbf{e}_{1}, \quad t \in[0,3]
$$

and

$$
u_{2}(t)=\left\{\begin{array}{lc}
\mathbf{e}_{2}, & t \in[0,1] \\
\frac{1}{2} \mathbf{e}_{1}, & t \in(1,2] \\
0, & t \in(2,3] .
\end{array}\right.
$$

Then both $x_{1}$ and $x_{2}$ are in $\partial \mathcal{A}(\mathcal{K}, t)$ for every $t \in[0,3]$, but "morally" only $x_{1}$ is really extremal. Indeed, the point $y=x_{2}(2)=x_{2}(3)$ is reached in time $\sqrt{3} / \sqrt{2}$ by the trajectory $x_{3}(\cdot)$ associated to the control

$$
u_{3}(t)=\frac{1}{2} \mathbf{e}_{1}+\frac{\sqrt{3}}{2} \mathbf{e}_{2} .
$$

On the other hand, the point $z=x_{1}(3)$ cannot be reached in any time $t<3$.

Definition 2.1. A solution $x(\cdot)$ of control system (4) is an optimal trajectory (or extremal solution) on $[0, T]$ if $\tau_{\mathcal{K}}(x(t))=t$ for all $t \in[0, T]$. We denote the set of optimal trajectories on $[0, T]$ by $\mathcal{X}(T)$.

Note that the relation $\tau_{\mathcal{K}}(x(T))=T$ suffices to guarantee that, for all $t$ in $[0, T]$, we also have $\tau_{\mathcal{K}}(x(t))=t$.

Now, we can focus our attention on the Maximum Principle. Define the Hamiltonian function as

$$
H(t, x, p)=\sup _{u \in U}\langle f(t, x, u), p\rangle \quad \forall(t, x, p) \in \mathbb{R}_{+} \times \mathbb{R}^{n} \times \mathbb{R}^{n} .
$$

Proposition 2.1. If $x(\cdot)$ is an optimal trajectory on $[0, T]$, then there exist an arc $p(\cdot)$, with $|p(0)|>0$, and a Radon measure $\mu$ such that for a.e. $t \in[0, T]$

$$
\left(h^{\prime}(t),-p^{\prime}(t), x^{\prime}(t)\right) \in \partial H(t, x(t), p(t)+\psi(t)),
$$

where $\psi(t)=\int_{[0, t)} \operatorname{Dd}(x(s)) \mu(d s)$ and $h(t)=H(t, x(t), p(t)+\psi(t))$. Here, measure $\mu$ is supported only by the set of times $t$ for which $x(t) \in \partial \Omega$. 
For a proof, see Ref. 7. In general, we don't know if $\psi$ (hence $h$ ) is continuous. To handle this boundary term, we need some regularity of the Hamiltonian. We will assume that

$$
\left\{\begin{array}{l}
H \text { is of class } \mathcal{C}^{2} \text { on } \mathbb{R}_{+} \times \mathbb{R}^{n} \times\left(\mathbb{R}^{n} \backslash\{0\}\right) \\
M|p| \geq H(t, x, p) \geq \alpha|p| \\
\Gamma I_{n} \geq D_{p p}^{2} H^{2}(t, x, p) \geq \gamma I_{n}
\end{array}\right.
$$

for some constants $\alpha, \gamma, \Gamma, M>0$, and for all $(t, x, p) \in \mathbb{R}_{+} \times \mathbb{R}^{n} \times\left(\mathbb{R}^{n} \backslash\{0\}\right)$.

Remark 2.1. Note that the last assumption of (7) is made for $H^{2}$, and not for $H$. In fact, such an assumption, if imposed on $H$, would be too restrictive (see example 2.2).

Moreover, we will impose the following growth conditions for the derivatives of $H$ : for all $(t, x, p) \in \mathbb{R}_{+} \times \mathbb{R}^{n} \times\left(\mathbb{R}^{n} \backslash\{0\}\right)$,

$$
\left\{\begin{array}{l}
\left|D_{t} H(t, x, p)\right| \leq M|p| \\
\left|D_{x} H(t, x, p)\right| \leq M|p| \\
\left|D_{p} H(t, x, p)\right| \leq M \\
\left|D_{p t}^{2} H(t, x, p)\right| \leq M \\
\left\|D_{p x}^{2} H(t, x, p)\right\| \leq M .
\end{array}\right.
$$

It is not restrictive to consider the same constant $M$ of (7).

Remark 2.2. We can give sufficient conditions for $f$ to satisfy some of the assumptions in (8). If we assume that, for all $(t, x, u) \in \mathbb{R}_{+} \times \mathbb{R}^{n} \times U$, we have $|f(t, x, u)| \leq M$ and $\left|D_{t} f(t, x, u)\right| \leq M$ and $\left\|D_{x} f(t, x, u)\right\| \leq M$, then the first three bounds in (8) are satisfied.

Moreover, if $\{f(t, x, U)\}_{(t, x) \in \mathbb{R}_{+} \times \mathbb{R}^{n}}$ is a family of uniformly convex sets of class $\mathcal{C}^{2}$, then $H \in \mathcal{C}^{2}\left(\mathbb{R}_{+} \times \mathbb{R}^{n} \times\left(\mathbb{R}^{n} \backslash\{0\}\right)\right)$, see, e.g., Ref. 5 .

Under the above regularity assumptions for the Hamiltonian, the Maximum Principle takes the more precise form described below.

Theorem 2.1. Assume that (3), (5), (7) and (8) hold true. Let $x(\cdot)$ be an optimal trajectory on the time interval $[0, T]$. Then there exists a Lipschitz continuous arc $p:[0, T] \rightarrow \mathbb{R}^{n}$, with $|p(t)|>0$, and a bounded measurable function $\lambda(t) \geq 0$ such that

$$
\left\{\begin{array}{l}
x^{\prime}(t)=D_{p} H(t, x(t), p(t)) \quad \text { for all } t \in[0, T], \\
-p^{\prime}(t)=D_{x} H(t, x(t), p(t))-\lambda(t) D d(x(t)) \quad \text { for a.e. } t \in[0, T] .
\end{array}\right.
$$


Moreover, $\lambda$ is the function given below:

$$
\begin{aligned}
\lambda=\frac{1}{\left\langle D_{p p}^{2} H(t, x, p) D d(x), D d(x)\right\rangle} & {\left[\left\langle D_{p p}^{2} H(t, x, p) D_{x} H(t, x, p), D d(x)\right\rangle\right.} \\
& -\left\langle D^{2} d(x) D_{p} H(t, x, p), D_{p} H(t, x, p)\right\rangle \\
& -\left\langle D_{x p}^{2} H(t, x, p) D_{p} H(t, x, p), D d(x)\right\rangle \\
& \left.-\left\langle D_{p t}^{2} H(t, x, p), D d(x)\right\rangle\right] \mathbf{1}_{\partial \Omega}(x),
\end{aligned}
$$

where the term $\left\langle D_{p p}^{2} H(t, x, p) D d(x), D d(x)\right\rangle$ is bounded from below by a positive constant on the set $\{t \in[0, T]: x(t) \in \partial \Omega\}$.

Proof. The idea of the proof is to approximate system (4) by a penalized control system without state constraints. Then we will apply the nonsmooth Maximum Principle to such a system. Finally, we will retrieve useful information for the original system (4).

Let $\varepsilon<\eta / 2$ be a positive fixed constant (where $\eta$ is defined in (3)), let

$$
f_{\varepsilon}(t, x, u):=f(t, x, u)\left(1-\frac{1}{\varepsilon} d_{\Omega}(x)\right)_{+},
$$

and consider the unconstrained system

$$
\left\{\begin{array}{l}
x^{\prime}(t)=f_{\varepsilon}(t, x(t), u(t)), \quad \text { a.e. } t \geq 0 \\
x(0)=x_{0} .
\end{array}\right.
$$

The associated perturbed Hamiltonian and the set of optimal trajectories are denoted as follows

$$
\begin{aligned}
H_{\varepsilon}(t, x, p) & =\max _{u \in U}\left\langle f_{\varepsilon}(t, x, u), p\right\rangle \quad \forall(t, x, p) \in \mathbb{R}_{+} \times \mathbb{R}^{n} \times \mathbb{R}^{n}, \\
\mathcal{X}_{\varepsilon}(T) & =\{\text { optimal trajectories of system (9) on }[0, T]\} .
\end{aligned}
$$

We note that $f_{\varepsilon}$ is Lipschitz continuous, but nonsmooth on the boundary of $\Omega$, and that

$$
H_{\varepsilon}(t, x, p)=H(t, x, p)\left(1-\frac{1}{\varepsilon} d_{\Omega}(x)\right)_{+} .
$$

We shall prove that, as soon as $\varepsilon$ is small enough, any trajectory of $\mathcal{X}(T)$ is actually a trajectory of $\mathcal{X}_{\varepsilon}(T)$, i.e. $\mathcal{X}(T) \subseteq \mathcal{X}_{\varepsilon}(T)$. This is not an obvious fact. In fact, it is clear that any constrained solution of (4) is still a solution of system (9), but an optimal trajectory $x(\cdot) \in \mathcal{X}(T)$ may fail to be optimal for (9). Indeed, system (9) can have more trajectories that arrive at the point $x(T) \in \bar{\Omega}$. 
126

We split the proof in 3 steps. The first and second step are devoted to show that $\mathcal{X}(T) \subseteq \mathcal{X}_{\varepsilon}(T)$ (so that we can use the nonsmooth Maximum Principle). In the third step we use the nonsmooth Maximum Principle to recover the conclusion in the constrained case.

Step 1 Let us check that, for a suitable choice of $\varepsilon$, any optimal trajectory of system (9) that stays in $\bar{\Omega}$ at time $T>0$, remains in $\bar{\Omega}$ for all $t<T$. Equivalently, we want to prove that

$$
\mathcal{X}_{\varepsilon}^{\bar{\Omega}}(T):=\left\{x(\cdot) \in \mathcal{X}_{\varepsilon}(T): x(T) \in \bar{\Omega}\right\} \subseteq \mathcal{T}_{\text {ad }}(\mathcal{K}, T) .
$$

Take $x_{\varepsilon}(\cdot) \in \mathcal{X}_{\varepsilon}^{\bar{\Omega}}(T)$. By the definition of $f_{\varepsilon}$,

$$
d_{\Omega}\left(x_{\varepsilon}(t)\right)<\varepsilon<\frac{\eta}{2} \quad \forall t \in[0, T] .
$$

Since $x_{\varepsilon}(\cdot)$ is optimal for an unconstrained problem, we can use the Maximum Principle of proposition 2.1 for nonsmooth hamiltonians without the boundary term $\psi(t)$. So, we find that there is some adjoint map $p_{\varepsilon}:[0, T] \rightarrow \mathbb{R}^{n}$ such that, for a.e. $t \in[0, T]$,

$$
\left\{\begin{array}{l}
x_{\varepsilon}^{\prime}(t)=D_{p} H\left(t, x_{\varepsilon}(t), p_{\varepsilon}(t)\right)\left(1-\frac{1}{\varepsilon} d_{\Omega}\left(x_{\varepsilon}(t)\right)\right)_{+} \\
-p_{\varepsilon}^{\prime}(t) \in \partial_{x} H_{\varepsilon}\left(t, x_{\varepsilon}(t), p_{\varepsilon}(t)\right) .
\end{array}\right.
$$

We observe that, for each $t$ such that $x_{\varepsilon}(t) \notin \partial \Omega$, the generalized gradient $\partial_{x} H_{\varepsilon}\left(t, x_{\varepsilon}(t), p_{\varepsilon}(t)\right)$ reduces to a singleton and

$$
\begin{aligned}
-p_{\varepsilon}^{\prime}(t)= & D_{x} H\left(t, x_{\varepsilon}(t), p_{\varepsilon}(t)\right)\left(1-\frac{1}{\varepsilon} d_{\Omega}\left(x_{\varepsilon}(t)\right)\right)+ \\
& -\frac{\lambda_{\varepsilon}(t) H\left(t, x_{\varepsilon}(t), p_{\varepsilon}(t)\right)}{\varepsilon} \operatorname{Dd}\left(x_{\varepsilon}(t)\right),
\end{aligned}
$$

where

$$
\begin{cases}\lambda_{\varepsilon}(t)=0 & \text { if } x_{\varepsilon}(t) \in \Omega \\ \lambda_{\varepsilon}(t)=1 & \text { if } x_{\varepsilon}(t) \notin \bar{\Omega}\end{cases}
$$

Note that $\left|p_{\varepsilon}(t)\right|>0$ for all $t \in[0, T]$. In fact we can normalize $p_{\varepsilon}$ so that $\left|p_{\varepsilon}(0)\right|=1$. Moreover, setting $h_{\varepsilon}(t)=H_{\varepsilon}\left(t, x_{\varepsilon}(t), p_{\varepsilon}(t)\right)$, we have that

$$
h_{\varepsilon}^{\prime}(t)=D_{t} H_{\varepsilon}\left(t, x_{\varepsilon}(t), p_{\varepsilon}(t)\right) \geq-M\left|p_{\varepsilon}(t)\right|\left(1-\frac{d_{\Omega}\left(x_{\varepsilon}(t)\right)}{\varepsilon}\right)_{+},
$$

thanks to (6) and (8). We recall that, in view of $(7), H_{\varepsilon}(t, x, p) \geq \alpha|p|(1-$ $\left.d_{\Omega}(x) / \varepsilon\right)_{+}$. Applying Gronwall's lemma we get $h_{\varepsilon}(t) \geq e^{-(M / \alpha) t} h_{\varepsilon}(0)$, and, since $H\left(t, x_{\varepsilon}(t), p_{\varepsilon}(t)\right) \geq H_{\varepsilon}\left(t, x_{\varepsilon}(t), p_{\varepsilon}(t)\right)$,

$$
H\left(t, x_{\varepsilon}(t), p_{\varepsilon}(t)\right) \geq e^{-\frac{M}{\alpha} t} H\left(0, x_{\varepsilon}(0), p_{\varepsilon}(0)\right) \geq e^{-\frac{M}{\alpha} t} \alpha>0 .
$$


In particular, this implies that $\left|p_{\varepsilon}(t)\right|>0$ on $[0, T]$.

Now, in order to conclude the proof of step 1, suppose, by contradiction, that there exists an interval $(a, b)$ on which $x_{\varepsilon}(t) \notin \bar{\Omega}$, and $x_{\varepsilon}(a), x_{\varepsilon}(b) \in$ $\partial \Omega$. Then, using right and left derivative of $t \mapsto d\left(x_{\varepsilon}(t)\right)$ in, respectively, $a$ and $b$, we obtain

$$
\left\{\begin{array}{l}
\left.\frac{d^{+}}{d t^{+}} d\left(x_{\varepsilon}(t)\right)\right|_{t=a}=\left\langle D_{p} H\left(a, x_{\varepsilon}(a), p_{\varepsilon}(a)\right), D d\left(x_{\varepsilon}(a)\right)\right\rangle \geq 0 \text { and } \\
\left.\frac{d^{-}}{d t^{-}} d\left(x_{\varepsilon}(t)\right)\right|_{t=b}=\left\langle D_{p} H\left(b, x_{\varepsilon}(b), p_{\varepsilon}(b)\right), D d\left(x_{\varepsilon}(b)\right)\right\rangle \leq 0 .
\end{array}\right.
$$

Since $H$ is nonnegative, these inequalities can also be rewritten as

$$
\left\{\begin{array}{l}
\left\langle D_{p} H^{2}\left(a, x_{\varepsilon}(a), p_{\varepsilon}(a)\right), D d\left(x_{\varepsilon}(a)\right)\right\rangle \geq 0 \text { and } \\
\left\langle D_{p} H^{2}\left(b, x_{\varepsilon}(b), p_{\varepsilon}(b)\right), \operatorname{Dd}\left(x_{\varepsilon}(b)\right)\right\rangle \leq 0 .
\end{array}\right.
$$

Observe that there exists a constant $C$ (not depending on $\varepsilon$ ) such that, for all $t \in[a, b]$,

$$
\left\|D^{2} d\left(x_{\varepsilon}(t)\right)\right\| \leq C,
$$

since, thanks to $(10), x_{\varepsilon}(t)$ is in a set in which $d(\cdot)$ is of class $\mathcal{C}_{\mathrm{b}}^{2}$. Hence

$$
\begin{aligned}
0 \geq & \int_{a}^{b} \frac{d}{d t}\left\langle D_{p} H^{2}\left(t, x_{\varepsilon}(t), p_{\varepsilon}(t)\right), D d\left(x_{\varepsilon}(t)\right)\right\rangle d t \\
= & \int_{a}^{b}\left\langle D_{p t}^{2} H^{2}\left(t, x_{\varepsilon}, p_{\varepsilon}\right)+D_{x p}^{2} H^{2}\left(t, x_{\varepsilon}, p_{\varepsilon}\right) x_{\varepsilon}^{\prime}+D_{p p}^{2} H^{2}\left(t, x_{\varepsilon}, p_{\varepsilon}\right) p_{\varepsilon}^{\prime}, D d\left(x_{\varepsilon}\right)\right\rangle d t \\
& +\int_{a}^{b}\left\langle D_{p} H^{2}\left(t, x_{\varepsilon}, p_{\varepsilon}\right), D^{2} d\left(x_{\varepsilon}\right) x_{\varepsilon}^{\prime}\right\rangle d t \\
\geq & -\int_{a}^{b}\left\|D_{x p}^{2} H^{2}\left(t, x_{\varepsilon}, p_{\varepsilon}\right)\right\|\left|f\left(t, x_{\varepsilon}, u\right)\right| d t \\
& -\int_{a}^{b}\left(\left\|D_{p p}^{2} H^{2}\left(t, x_{\varepsilon}, p_{\varepsilon}\right)\right\|\left|D_{x} H\left(t, x_{\varepsilon}, p_{\varepsilon}\right)\right|+\left|D_{p t}^{2} H^{2}\left(t, x_{\varepsilon}, p_{\varepsilon}\right)\right|\right) d t \\
& -\int_{a}^{b}\left|D_{p} H^{2}\left(t, x_{\varepsilon}, p_{\varepsilon}\right)\right|\left\|D^{2} d\left(x_{\varepsilon}\right)\right\|\left|f\left(t, x_{\varepsilon}, u\right)\right| d t \\
& +\int_{a}^{b} \frac{H\left(t, x_{\varepsilon}, p_{\varepsilon}\right)}{\varepsilon}\left\langle D_{p p}^{2} H^{2}\left(t, x_{\varepsilon}, p_{\varepsilon}\right) D d\left(x_{\varepsilon}\right), D d\left(x_{\varepsilon}\right)\right\rangle d t \\
\geq & \int_{a}^{b}\left(\frac{H\left(t, x_{\varepsilon}, p_{\varepsilon}\right)}{\varepsilon} \gamma-M^{\prime}\left(1+\left|p_{\varepsilon}\right|\right)\right) d t
\end{aligned}
$$

where $\gamma$ is defined by $(7)$ and $M^{\prime}=2 M^{3}(2+C)+4 M^{2}+\Gamma M$.

Finally, we get a contradiction setting

$$
\varepsilon:=\frac{\alpha \gamma e^{-\frac{M}{\alpha} T}}{4 M^{\prime}} .
$$


Indeed $H\left(t, x_{\varepsilon}, p_{\varepsilon}\right) \gamma / \varepsilon-M^{\prime}\left(1+\left|p_{\varepsilon}\right|\right)>0$ if, for $\left|p_{\varepsilon}\right| \leq 1$, we choose $\varepsilon<$ $\alpha \gamma e^{-(M / \alpha) T} /\left(2 M^{\prime}\right)$, and, for $\left|p_{\varepsilon}\right|>1$, we choose $\varepsilon<\alpha \gamma /\left(2 M^{\prime}\right)$.

Step 2 We want to show that an extremal solution of the constrained problem (4) is also an extremal solution of (9), i.e., that $\mathcal{X}(T) \subseteq \mathcal{X}_{\varepsilon}(T)$.

Let $x(\cdot) \in \mathcal{X}(T)$, and let $T_{\varepsilon}$ the minimum time to reach the point $x(T)$ for the perturbed problem (9). Since any solution of the constrained problem (4) is also a solution of the unconstrained problem (because $f_{\left.\varepsilon\right|_{\Omega}}=$ $\left.f_{\left.\right|_{\Omega}}\right)$, we have that $T_{\varepsilon} \leq T$.

Now, let $x_{\varepsilon}(\cdot)$ be a trajectory of the perturbed problem (9) such that $x_{\varepsilon}\left(T_{\varepsilon}\right)=x(T)$. Then $x_{\varepsilon}(\cdot)$ is in $\mathcal{X}_{\varepsilon}^{\bar{\Omega}}\left(T_{\varepsilon}\right)$, and, by step 1 , we know that it is also a solution of the original problem (4), as it ends in $\bar{\Omega}$. Then $T_{\varepsilon} \geq T$, and we have that $T_{\varepsilon}=T$ and $x(\cdot) \in \mathcal{X}_{\varepsilon}(T)$.

Step 3 Finally, let $x(\cdot) \in \mathcal{X}(T)$ and $p(\cdot)$ be an associated adjoint map such that $(x(\cdot), p(\cdot))$ satisfies $(11)$. We want to find an explicit expression for $p^{\prime}(t)$. For this we define the set of times $t$ for which the trajectory $x(\cdot)$ stays on the boundary, that is

$$
E_{x}=\{t \in[0, T]: x(t) \in \partial \Omega\} .
$$

Observe that, by standard properties of the generalized gradient,

$$
\partial_{x} H_{\varepsilon}(t, x, p) \subseteq \partial_{x} H(t, x, p)\left(1-\frac{d_{\Omega}(x)}{\varepsilon}\right)_{+}+H(t, x, p) \partial_{x}\left(1-\frac{d_{\Omega}(x)}{\varepsilon}\right)_{+}
$$

for all $x \in \partial \Omega$ (see Ref. 7). Moreover, for all $x \in \partial \Omega$,

$$
\partial_{x} H(t, x, p)=D_{x} H(t, x, p), \quad \partial_{x}\left(1-\frac{d_{\Omega}(x)}{\varepsilon}\right)=-\operatorname{Dd}(x)\left[0, \frac{1}{\varepsilon}\right] .
$$

Recalling (11) and (12), we have that there is a measurable function $\lambda_{\varepsilon}$ : $[0, T] \rightarrow[0,1]$ such that, for a.e. $t \in[0, T]$,

$$
-p^{\prime}(t)=D_{x} H(t, x(t), p(t))-\frac{\lambda_{\varepsilon}(t) H(t, x(t), p(t))}{\varepsilon} D d(x(t)),
$$

where $\lambda_{\varepsilon}(t)$ is given by (13) for $t \notin E_{x}$. Now define the function

$$
\varphi(t):=d(x(t)) \quad t \in[0, T] .
$$

Since $\Omega$ is of class $\mathcal{C}^{2}$ and $x^{\prime}(t)$ exists for a.e. $t$, we have that $\varphi$ is differentiable for a.e. $t \in E_{x}$. Moreover, for a.e. $t \in E_{x}$,

$$
\left\langle D d(x(t)), D_{p} H(t, x(t), p(t))\right\rangle=\left\langle D d(x(t)), x^{\prime}(t)\right\rangle=\varphi^{\prime}(t)=0 .
$$

In fact, let $t \in E_{x}$. If $\varphi^{\prime}(t)>0$ then, by continuity, there exists some $\eta>0$ such that $\varphi(s)>0$ for all $s \in(t, t+\eta)$, in contrast to $x(t) \in \bar{\Omega}$ 
for all $t \in[0, T]$. Similarly, if $\varphi^{\prime}(t)<0$ we will have that $\varphi(s)>0$ for all $s \in(t-\eta, t)$.

In $E_{x}$, we can differentiate (18) with respect to $t$. We obtain

$$
\begin{aligned}
0= & \left\langle D^{2} d(x(t)) x^{\prime}(t), D_{p} H(t, x(t), p(t))\right\rangle+\left\langle D d(x(t)), D_{p t}^{2} H(t, x(t), p(t))\right\rangle \\
& +\left\langle D d(x(t)), D_{x p}^{2} H(t, x(t), p(t)) x^{\prime}(t)\right\rangle \\
& +\left\langle D d(x(t)), D_{p p}^{2} H(t, x(t), p(t)) p^{\prime}(t)\right\rangle .
\end{aligned}
$$

We focus our attention on the last term of this equation, seeing that

$$
\begin{aligned}
\langle & \left.D d(x(t)), D_{p p}^{2} H(t, x(t), p(t)) p^{\prime}(t)\right\rangle= \\
= & \left\langle D d(x(t)), D_{p p}^{2} H(t, x(t), p(t))\left[H(t, x(t), p(t)) \frac{\lambda_{\varepsilon}(t)}{\varepsilon} D d(x(t))-D_{x} H(t, x(t), p(t))\right]\right\rangle \\
= & \frac{\lambda_{\varepsilon}(t)}{\varepsilon} H(t, x(t), p(t))\left\langle D d(x(t)), D_{p p}^{2} H(t, x(t), p(t)) D d(x(t))\right\rangle \\
& -\left\langle D d(x(t)), D_{p p}^{2} H(t, x(t), p(t)) D_{x} H(t, x(t), p(t))\right\rangle .
\end{aligned}
$$

We claim that, for $|p(t)|>0,\left\langle D d(x(t)), D_{p p}^{2} H(t, x(t), p(t)) D d(x(t))\right\rangle>0$.

Indeed, for a unit vector $\zeta \in \mathbb{R}^{n}$, we have that

$$
\begin{aligned}
H(t, x, p)\left\langle D_{p p}^{2} H(t, x, p) \zeta, \zeta\right\rangle & =\frac{\left\langle D_{p p}^{2} H^{2}(t, x, p) \zeta, \zeta\right\rangle}{2}-\left\langle D_{p} H(t, x, p), \zeta\right\rangle^{2} \\
& \geq \frac{\gamma}{2}-\left\langle D_{p} H(t, x, p), \zeta\right\rangle^{2}
\end{aligned}
$$

thanks to (7). Then,

$$
\left\langle\frac{D_{p} H(t, x, p)}{\left|D_{p} H(t, x, p)\right|}, \zeta\right\rangle^{2} \leq \frac{\gamma}{4 M^{2}} \quad \Longrightarrow \quad H(t, x, p)\left\langle D_{p p}^{2} H(t, x, p) \zeta, \zeta\right\rangle \geq \frac{\gamma}{4} .
$$

As a consequence of (18), there exists a constant $\delta>0$ such that

$$
\left\langle D d(x(t)), D_{p p}^{2} H(t, x(t), p(t)) D d(x(t))\right\rangle>\frac{\delta}{|p(t)|} \quad \text { a.e. } t \in E_{x} .
$$

Finally, recalling the expression of $p^{\prime}$ in (17), we find a representation of $\lambda$ by setting

$$
\lambda(t):=\frac{\lambda_{\varepsilon}(t)}{\varepsilon} H(t, x(t), p(t)) .
$$

Moreover, since

$$
e^{-M(1+1 / \varepsilon) T} \leq|p(t)| \leq e^{M(1+1 / \varepsilon) T},
$$

(where $\varepsilon$ is defined in (16)) we have that $\lambda$ is bounded and $p(\cdot)$ is Lipschitz continuous. Observing that the right-hand side of the equality 
$x^{\prime}=D_{p} H(t, x, p)$ is continuous, we conclude that this equality holds for all $t$ in $[0, T]$.

Example 2.2. We discuss a class of control systems that satisfy the assumptions of this section for the maximum principle. Consider the control system

$$
\left\{\begin{array}{l}
x^{\prime}(t)=f(t, x(t)) u(t), \quad u(t) \in \bar{B} \\
x(0)=x_{0} \in \mathcal{K},
\end{array}\right.
$$

where $f: \mathbb{R}_{+} \times \mathbb{R}^{n} \rightarrow M_{n}(\mathbb{R})$ is of class $\mathcal{C}^{2}$, and the matrix $f(t, x)$ is bounded and invertible, with bounded inverse.

Moreover, the Hamiltonian is

$$
H(t, x, p)=\max _{u \in \bar{B}_{1}}\langle f(t, x) u, p\rangle=\max _{u \in \bar{B}_{1}}\left\langle u, f^{*}(t, x) p\right\rangle=\left|f^{*}(t, x) p\right| .
$$

So, assumptions (7) are satisfied, since $f$ and $f^{-1}$ are bounded, and

$$
D_{p p}^{2} H^{2}(t, x, p)=2 f(t, x) f^{*}(t, x) .
$$

Since we can consider $(t, x)$ in a compact set, then $D_{t} f(t, x)$ and $D_{x} f(t, x)$ are bounded, and, thanks to remark 2.2, $D_{t} H(t, x, p)$ and $D_{x} H(t, x, p)$ satisfy assumptions (8).

Moreover,

$$
D_{p} H(t, x, p)=\frac{f(t, x) f^{*}(t, x) p}{\left|f^{*}(t, x) p\right|} .
$$

Then we have

$$
\left|D_{p t}^{2} H(t, x, p)\right| \leq 2\left\|D_{t} f(t, x)\right\|+\|f(t, x)\|\left\|f^{-1}(t, x)\right\|\left\|D_{t} f(t, x)\right\|,
$$

and

$$
\left\|D_{p x}^{2} H(t, x, p)\right\| \leq 2\left\|D_{x} f(t, x)\right\|+\|f(t, x)\|\left\|f^{-1}(t, x)\right\|\left\|D_{x} f(t, x)\right\| .
$$

Hence all the assumptions in (8) are satisfied.

Finally note that, for any vector $\zeta \in \mathbb{R}^{n}$,

$$
\left\langle D_{p p}^{2} H(t, x, p) \zeta, \zeta\right\rangle=\frac{1}{\left|f^{*}(t, x) p\right|}\left(\left|f^{*}(t, x) \zeta\right|^{2}-\left\langle\frac{f^{*}(t, x) p}{\left|f^{*}(t, x) p\right|}, f^{*}(t, x) \zeta\right\rangle^{2}\right)
$$

so that it would be impossible to satisfy $\Gamma I_{n} \geq D_{p p}^{2} H(t, x, p) \geq \gamma I_{n}$, even for this simple control system. 


\section{Perimeter estimates for the attainable set}

In this section we will study the special case of control systems in $\mathbb{R}^{2}$ of the form

$$
\left\{\begin{array}{l}
x^{\prime}(t)=f(t, x(t)) u(t), \quad \text { a.e. } t \geq 0 \\
x(t) \in \bar{\Omega}, \quad t \geq 0 \\
x(0)=x_{0} \in \mathcal{K},
\end{array}\right.
$$

where $u: \mathbb{R}_{+} \rightarrow \bar{B}$ is a measurable function, and $f: \mathbb{R}_{+} \times \mathbb{R}^{2} \rightarrow M_{2}(\mathbb{R})$ is such that

- $f$ is of class $\mathcal{C}^{2}$ in $\mathbb{R}_{+} \times \bar{\Omega}$

- $f(t, x)$ is invertible for any $(t, x) \in \mathbb{R}_{+} \times \bar{\Omega}$

- $f(t, x)$ and $f^{-1}(t, x)$ are bounded by $M>0$ for any $(t, x) \in \mathbb{R}_{+} \times \bar{\Omega}$.

The Hamiltonian for this dynamic is

$$
H(t, x, p)=\max _{u \in \bar{B}}\langle f(t, x) u, p\rangle=\left|f^{*}(t, x) p\right| .
$$

The aim of this section is to estimate the perimeter of the attainable set

$$
\mathcal{A}(t):=\mathcal{A}(\mathcal{K}, t) .
$$

Cardaliaguet and $\mathrm{Marchi}^{6}$ proved such an estimate for $f(t, x)=c(t, x) I_{n}$, where $c$ is a scalar function. Applying theorem 2.1 we can use their technique to extend this analysis to system (20).

Theorem 3.1. Let $T>0$ be fixed. For any $t \in(0, T]$ the attainable set $\mathcal{A}(t)$ is of finite perimeter, and there exist $C_{1}, C_{2}>0$ such that

$$
\mathcal{H}^{1}(\partial \mathcal{A}(t)) \leq C_{1}\left(\mathcal{H}^{1}(\partial \Omega)+\frac{e^{C_{2} t}}{t}\right) .
$$

In particular, if set $\mathcal{K}$ has the interior sphere property, then the attainable set $\mathcal{A}(t)$ has finite perimeter for any $t \in[0, T]$, and the perimeter of $\mathcal{A}(t)$ is bounded on $[0, T]$.

In order to prove that $\mathcal{A}(t)$ has finite perimeter, we cover the boundary of the attainable set with the following sets

$\mathcal{B}^{\text {bnd }}(t):=\{x \in \bar{\Omega}: \exists x(\cdot) \in \mathcal{X}(t), \exists s \in[0, t]$, with $x(t)=x, x(s) \in \partial \Omega\}$, $\mathcal{B}^{\text {int }}(t):=\{x \in \Omega: \exists x(\cdot) \in \mathcal{X}(t)$, with $x(t)=x$ and $x([0, t]) \cap \partial \Omega=\emptyset\}$.

Note that $\mathcal{B}^{\text {int }}(t)$ is the set of points that can be reached by extremal trajectories contained in the interior of $\Omega$, while $\mathcal{B}^{\text {bnd }}(t)$ is the set of points reached by extremal trajectories that touch the boundary $\partial \Omega$. Then

$$
\partial \mathcal{A}(t) \subseteq \mathcal{B}^{\text {int }}(t) \cup \mathcal{B}^{\text {bnd }}(t) \cup \partial \Omega .
$$


We point out that, in this case, the adjoint system of theorem 2.1 is given by

$$
-p^{\prime}(t)=|p(t)|\left(D_{x} H\left(t, x(t), \frac{p(t)}{|p(t)|}\right)-\bar{\lambda}(t) D d(x(t))\right),
$$

where $\bar{\lambda}$ is a bounded positive function.

Consequently, for all $T>0$, we have that the set $\mathcal{X}(T)$ of the extremal trajectories for system $(20)$ is compact with respect to the $\mathcal{C}^{1}$ norm.

With an easy adaptation we can recover from Ref. 6 a Lipschitz estimate for the velocities of extremal trajectories on the boundary of $\Omega$.

Lemma 3.1 (Ref. 6, lemma 4.1 ). Let $T$ and $\delta$ be given positive numbers, let $t_{1}, t_{2} \in(0, T]$, and let

$$
y_{i}:\left[0, t_{i}+\delta\right] \rightarrow \mathbb{R}^{2} \quad(i=1,2)
$$

be optimal trajectories for (20). There is a positive number $\sigma$ such that, if $y_{i}\left(t_{i}\right) \in \partial \Omega(i=1,2)$ and $\left|y_{1}\left(t_{1}\right)-y_{2}\left(t_{2}\right)\right| \leq \sigma$, then

$$
\left|\frac{y_{1}^{\prime}\left(t_{1}\right)}{\left|y_{1}^{\prime}\left(t_{1}\right)\right|}-\frac{y_{2}^{\prime}\left(t_{2}\right)}{\left|y_{2}^{\prime}\left(t_{2}\right)\right|}\right| \leq C_{\Omega}\left|y_{1}\left(t_{1}\right)-y_{2}\left(t_{2}\right)\right|
$$

where $C_{\Omega}$ is a positive constant depending only on the regularity of $\partial \Omega$.

In order to estimate $\mathcal{H}^{1}\left(\mathcal{B}^{\text {bnd }}(t)\right)$ the main idea is to use the perimeter of $\partial \Omega$. At this aim we define the set

$$
\mathcal{B}^{\text {bnd }}(t, \delta):=\left\{x \in \mathcal{B}^{\text {bnd }}(t): x((t-\delta, t]) \cap \partial \Omega=\emptyset\right\} .
$$

Lemma 3.2. Let $T>0$ be fixed, let $t \in(0, T]$, and let $y_{1}, y_{2} \in \mathcal{B}^{\mathrm{bnd}}(t, \delta)$. For $i=1,2$, let $y_{i}(\cdot) \in \mathcal{X}(t)$ and let $s_{i} \in[0, t-\delta)$ such that

$$
y_{i}(t)=y_{i}, \quad y_{i}\left(s_{i}\right) \in \partial \Omega, \quad y_{i}\left(\left(s_{i}, t\right]\right) \cap \partial \Omega=\emptyset .
$$

There is a constant $C_{0}>0$ such that, for any $\delta>0$, a constant $\sigma_{\delta}>0$ exists, so that, if

$$
\left|y_{1}\left(s_{1}\right)-y_{2}\left(s_{2}\right)\right| \leq \sigma_{\delta},
$$

then

$$
\left|y_{1}-y_{2}\right| \leq C_{0}\left|y_{1}\left(s_{1}\right)-y_{2}\left(s_{2}\right)\right| .
$$

Proof. Without loss of generality, we can suppose $s_{1} \leq s_{2}$. There exist adjoint states $p_{i}(\cdot)$ such that, for $s \geq s_{i}$ the extremal trajectories $y_{i}(\cdot)$ solve

$$
\left\{\begin{array}{l}
y_{i}^{\prime}(s)=D_{p} H\left(s, y_{i}(s), p_{i}(s)\right) \\
-p_{i}^{\prime}(s)=\left|p_{i}(s)\right| D_{x} H\left(s, y_{i}(s), \frac{p_{i}(s)}{\left|p_{i}(s)\right|}\right) .
\end{array}\right.
$$


We normalize $\operatorname{arcs} p_{i}(\cdot)$ so that

$$
H\left(s_{i}, y_{i}\left(s_{i}\right), p_{i}\left(s_{i}\right)\right)=\left|f^{*}\left(s_{i}, y_{i}\left(s_{i}\right)\right) p_{i}\left(s_{i}\right)\right|=1, \quad i=1,2 .
$$

Applying Gronwall's lemma we have

$$
\frac{1}{M} e^{-L\left(s-s_{i}\right)} \leq\left|p_{i}(s)\right| \leq M e^{L\left(s-s_{i}\right)} \quad \forall s \in\left[s_{i}, t\right] .
$$

Moreover, thanks to system (22), for any $s \in\left[s_{2}, t\right]$,

$$
\begin{array}{r}
\left|y_{1}(s)-y_{2}(s)\right| \leq\left|y_{1}(s)-y_{2}(s)\right|+\left|p_{1}(s)-p_{2}(s)\right| \\
\leq C\left(\left|y_{1}\left(s_{2}\right)-y_{2}\left(s_{2}\right)\right|+\left|p_{1}\left(s_{2}\right)-p_{2}\left(s_{2}\right)\right|\right) .
\end{array}
$$

Now, we focus our attention on the right-hand side of this inequality. For the first term, we have that

$$
\begin{aligned}
\left|y_{1}\left(s_{2}\right)-y_{2}\left(s_{2}\right)\right| & \leq\left|y_{1}\left(s_{1}\right)-y_{2}\left(s_{2}\right)\right|+\left|y_{1}\left(s_{2}\right)-y_{1}\left(s_{1}\right)\right| \\
& \leq\left|y_{1}\left(s_{1}\right)-y_{2}\left(s_{2}\right)\right|+M\left|s_{1}-s_{2}\right| \\
& \leq\left(1+M L_{0}\right)\left|y_{1}\left(s_{1}\right)-y_{2}\left(s_{2}\right)\right|,
\end{aligned}
$$

invoking the Lipschitz continuity (of rank $L_{0}$ ) of the minimum time function. Similarly, we have that

$$
\begin{aligned}
\left|p_{1}\left(s_{2}\right)-p_{2}\left(s_{2}\right)\right| & \leq\left|p_{1}\left(s_{1}\right)-p_{2}\left(s_{2}\right)\right|+\left|p_{1}\left(s_{2}\right)-p_{1}\left(s_{1}\right)\right| \\
& \leq\left|p_{1}\left(s_{1}\right)-p_{2}\left(s_{2}\right)\right|+C^{\prime}\left|y_{1}\left(s_{1}\right)-y_{2}\left(s_{2}\right)\right| .
\end{aligned}
$$

Recalling the expression of $D_{p} H(t, x, p)$ in (19), we have that

$$
\begin{aligned}
\left|p_{1}\left(s_{1}\right)-p_{2}\left(s_{2}\right)\right|= & \mid \overbrace{\left|f^{*}\left(s_{1}, y_{1}\left(s_{1}\right)\right) p_{1}\left(s_{1}\right)\right|}^{=1}\left[f\left(s_{1}, y_{1}\left(s_{1}\right)\right) f^{*}\left(s_{1}, y_{1}\left(s_{1}\right)\right)\right]^{-1} y_{1}^{\prime}\left(s_{1}\right) \\
& -\overbrace{\left|f^{*}\left(s_{2}, y_{2}\left(s_{2}\right)\right) p_{2}\left(s_{2}\right)\right|}^{=1}\left[f\left(s_{2}, y_{2}\left(s_{2}\right)\right) f^{*}\left(s_{2}, y_{2}\left(s_{2}\right)\right)\right]^{-1} y_{2}^{\prime}\left(s_{2}\right) \mid .
\end{aligned}
$$

Moreover, we also have that

$$
\begin{aligned}
& \left|y_{1}^{\prime}\left(s_{1}\right)-y_{2}^{\prime}\left(s_{2}\right)\right| \leq \\
& \quad \leq\left|y_{1}^{\prime}\left(s_{1}\right)\right|\left(\left|\frac{y_{1}^{\prime}\left(s_{1}\right)}{\left|y_{1}^{\prime}\left(s_{1}\right)\right|}-\frac{y_{2}^{\prime}\left(s_{2}\right)}{\left|y_{2}^{\prime}\left(s_{2}\right)\right|}\right|+\left|y_{2}^{\prime}\left(s_{2}\right)\right|\left|\frac{1}{\left|y_{1}^{\prime}\left(s_{1}\right)\right|}-\frac{1}{\left|y_{2}^{\prime}\left(s_{2}\right)\right|}\right|\right) .
\end{aligned}
$$

Since, for $i=1,2,\left|y_{i}^{\prime}\left(s_{i}\right)\right| y_{i}^{\prime}\left(s_{i}\right) /\left|y_{i}^{\prime}\left(s_{i}\right)\right|=f\left(s_{i}, y_{i}\left(s_{i}\right)\right) u_{i}\left(s_{i}\right)$, and $u_{i}\left(s_{i}\right) \in$ $\partial B$, we obtain that

$$
\left|\frac{1}{\left|y_{1}^{\prime}\left(s_{1}\right)\right|}-\frac{1}{\left|y_{2}^{\prime}\left(s_{2}\right)\right|}\right|=\left|f^{-1}\left(s_{1}, y_{1}\left(s_{1}\right)\right) \frac{y_{1}^{\prime}\left(s_{1}\right)}{\left|y_{1}^{\prime}\left(s_{1}\right)\right|}-f^{-1}\left(s_{2}, y_{2}\left(s_{2}\right)\right) \frac{y_{2}^{\prime}\left(s_{2}\right)}{\left|y_{2}^{\prime}\left(s_{2}\right)\right|}\right| \text {. }
$$

As a consequence of lemma 3.1 and of Lipschitz continuity of $f$ we have the conclusion. 
Thanks to lemma 3.2 we have a bound for the perimeter of $\mathcal{B}^{\text {bnd }}(t)$. We give a proof for the reader's convenience.

Proposition 3.1. Let $T>0$ be fixed. There is a constant $C_{0}>0$ such that, for all $t \in[0, T]$,

$$
\mathcal{H}^{1}\left(\mathcal{B}^{\text {bnd }}(t)\right) \leq C_{0} \mathcal{H}^{1}(\partial \Omega)
$$

Proof. For any fixed $\delta>0$, let $C_{0}$ and $\sigma_{\delta}$ the constants of lemma 3.2. Let $\varepsilon>0$, and let $\left\{B_{n}\right\}_{n \in \mathbb{N}}$ be a family of sets such that

$$
\partial \Omega \subseteq \bigcup_{n \in \mathbb{N}} B_{n}, \quad \mathcal{H}^{1}(\partial \Omega)+\varepsilon \geq \sum_{n=1}^{\infty} \operatorname{diam}\left(B_{n}\right), \quad \operatorname{diam}\left(B_{n}\right) \leq \inf \left\{\sigma_{\delta}, \varepsilon\right\} .
$$

Consider the covering of $\mathcal{B}^{\text {bnd }}(t, \delta)$, given by

$$
K_{n}=\left\{x \in \mathcal{B}^{\text {bnd }}(t, \delta): x(s) \in B_{n}, x((s, t]) \cap \partial \Omega=\emptyset\right\} .
$$

In view of lemma 3.2 , for all $n \in \mathbb{N}$, we have that

$$
\begin{aligned}
& \operatorname{diam}\left(K_{n}\right) \leq C_{0} \varepsilon \\
& \operatorname{diam}\left(K_{n}\right) \leq C_{0} \operatorname{diam}\left(B_{n}\right)
\end{aligned}
$$

Thus,

$$
\mathcal{H}_{C_{0} \varepsilon}^{1}\left(\mathcal{B}^{\text {bnd }}(t, \delta)\right) \leq \sum_{n=1}^{\infty} \operatorname{diam}\left(K_{n}\right) \leq C_{0}\left(\mathcal{H}^{1}(\partial \Omega)+\varepsilon\right)
$$

Now, letting $\varepsilon \rightarrow 0$ and then $\delta \rightarrow 0$, we have the desired result, since $C_{0}$ is independent of $\varepsilon$ and $\delta$.

Now, we turn our attention to set $\mathcal{B}^{\text {int }}(t)$. Following the main ideas of Ref. 3, we can see that this set has the interior ball property, and then it has finite perimeter for any $t>0$.

Remark 3.1. Let $x(\cdot)$ be an extremal solution on the interval $[0, T]$, such that $x([0, T]) \subseteq \Omega$. Then there exists some $\eta=\eta(x)>0$ such that, for all $s \in[0, T]$, we have that $B_{\eta}(x(s)) \subseteq \Omega$.

Proposition 3.2. Let $T>0$ be fixed. Then there exists a constant $c_{T}>0$ such that for any $x \in \mathcal{B}^{\operatorname{int}}(T)$, with $x(\cdot)$ extremal solution so that $x(T)=x$, then there exists some $\eta=\eta(x)>0$ such that

$$
B\left(x(t)-c_{T} t \frac{p(t)}{|p(t)|}, c_{T} t\right) \cap B\left(x(t), \eta \frac{e^{-L T}}{1+L T}\right) \subseteq \mathcal{A}(t) .
$$


From Ref. 1 and proposition 3.2, we can derive the following bound for the perimeter.

Proposition 3.3. Fix $T>0$. There exists $C>0$ such that

$$
\mathcal{H}^{1}\left(\mathcal{B}^{\text {int }}(t)\right) \leq \frac{C}{c_{T} t}
$$

The perimeter of $\mathcal{B}^{\text {int }}(t)$ is in inverse ratio to the time $t$, since the interior sphere property is proportional to the time. This means that, if we fix a time $\vartheta>0$, for any $t \geq \vartheta$ we have a uniform estimate (i.e. $C / c_{T} \vartheta$ ). In addition, if set $\mathcal{K}$ has the interior sphere property of radius $r$, then for all $t \in[0, T]$

$$
\mathcal{H}^{1}\left(\mathcal{B}^{\text {int }}(t)\right) \leq \frac{C}{r}
$$

\section{References}

1. O. Alvarez, P. Cardaliaguet, R. Monneau, Interf. Free Bound. 7, 415 (2005).

2. A.V. Arutyunov and S.M. Aseev, SIAM J. Control Optim. 35, 930 (1997).

3. P. Cannarsa and P. Cardaliaguet, J. Convex Anal. 13, 253 (2006).

4. P. Cannarsa and H. Frankowska, ESAIM Control Optim. Calc. Var. 12, 350 (2006).

5. P. Cannarsa and C. Sinestrari, Semiconcave functions, Hamilton-Jacobi equations and optimal control (Birkhäuser, Boston, 2004).

6. P. Cardaliaguet and C. Marchi, SIAM J. Control Optim. 45, 1017 (2006).

7. F.H. Clarke, Optimization and Nonsmooth analysis (Wiley-Interscience, New York, 1983).

8. A.Y. Dubovitskii and A.A. Milyutin, USSR Comput. Math. and Math. Physics 5, 1 (1965).

9. H. Frankowska, J. Convex Anal. 13, 299 (2006).

10. R.V. Gamkrelidze, Izk. Akad. Nauk., USSR Sec. Mat. 24, 315 (1960).

11. P. Loewen and R.T. Rockafellar, Trans. Amer. Math. Soc. 325, 39 (1991).

12. L.S. Pontryagin, V.G. Boltyanskii, R.V. Gamkrelidze, E.F. Mishchenko, The Mathematical theory of otpimal processes (Interscience Publishers John Wiley \& Sons Inc., New York-London, 1962).

13. R.B. Vinter, Optimal Control (Birkhäuser, Boston, Basel, Berlin, 2000). 